\title{
On the Set of Primitive Triples of Natural Numbers Satisfying the Diophantine Equation of Pythagor
}

\author{
Bagram Sibgatullovich Kochkarev \\ Department of Mathematics and Mathematical Modeling, Institute of Mathematics and Mechanics Named After Nikolai Ivanovich \\ Lobachevsky, Kazan (Volga Region) Federal University, Kazan, Russia
}

Email address:

bkochkarev@rambler.ru

\section{To cite this article:}

Bagram Sibgatullovich Kochkarev. On the Set of Primitive Triples of Natural Numbers Satisfying the Diophantine Equation of Pythagor. International Journal of Discrete Mathematics. Vol. 5, No. 1, 2020, pp. 1-3. doi: 10.11648/j.dmath.20200501.11

Received: October 31, 2019; Accepted: December 25, 2019; Published: April 23, 2020

\begin{abstract}
The main task considered in the article is to find the condition primitive integer solutions of the Diophantine Pithagorean equation $\mathrm{x}^{2}+\mathrm{y}^{2}=\mathrm{z}^{2}$ It is known that for this purpose it is enough to find primive solution of $\mathrm{x}$, $\mathrm{y}$ such that $\mathrm{x}$ is even and $\mathrm{y}$ is odd. In this paper, in particular, we proved that the $\mathrm{z}$ of a primitive solution is a Prime number of the form $4 \mathrm{k}+1$. It is prove in this paper that any right triangle with integer side lengths has a hypotenuse equal to a Prime of the form $4 \mathrm{k}+1$ and we show with the help of the descent axiom how to find primitive solutions of $\mathrm{x}$ and $\mathrm{y}$ in this case. We divide the search for primitive solutions $(\mathrm{x}, \mathrm{y}, \mathrm{z})$ of right triangles into two cases: 1) the hypotenuse of such triangles is a Prime number of the form $4 \mathrm{k}+1$ and 2 ) the hypotenuse of such triangles is a composite number. In section 3 we use formulas known to the ancient Hindus to find primitive solutions of Pithagorean equations in cases where $m$ and $n<m$ are mutually Prime numbers of different parity, and this happens in cases where, for example, $\mathrm{n}=4$, and $\mathrm{m}$ is a Prime number ending in 3 or 7 . In such cases, the hypotenuse $\mathrm{z}=\mathrm{m}^{2}+\mathrm{n}^{2}$ is an compaund number ending in 5. To find primes ending in 3 and 7, we refer the reader to our paper, which presents algorithms for constructing all primes and twin primes. The proposed paper also presents a generalization of Euclid's fundamental result on the infinity of the set of Primes, namely, it is shown that all twin primes are in residue classes $(1,3),(2,4),(4,1)$, and there are infinity many such twins.
\end{abstract}

Keywords: Prime Numbers, Binary Problem, Axiom of Descent

\section{Introduction}

First of all, let us recall [1] the mathematical definition of a binary statement from the natural parameter and axiom of descent.

Definition 1. A mathematical statement $A_{n}$ depending on natural parameter $n$ we will call binary if for any value $n=\alpha$ the statement $A_{\varepsilon}$ has one of the other values truth or lie.

Axiom of descent [1]: let $A_{n}$ will be the binary statement from natural parameter such that 1) there is an algorithm which for any value $n$ gives the answer on the question "statement $A_{n}$ truth or lie?" 2) for the values of parameter $n_{1}<n_{2}<\ldots<n_{k}$ the statements $A_{n_{1}}, A_{n_{2}}, \ldots, A_{n_{k}}$ are true, and for any $n>n_{k}$ the statement $A_{n}$ is false. Then the statement $A_{n}$ is true for infinity many of values $\mathrm{n}$.
Many important mathematical problems remained open for a time. We believe that this circumstance was due to the fact that the foundations of mathematics required a good revision. For example Euclidean geometry lasted firmly over 2000 years, and only appeared in the 19th century non-Eucldean geometry (Lobachevsky and Riemann) after review of axioms Euclid. The same find happens in other areas of mathematics. Especially many have accumulated open problems in numbers theory - the most ancient field of mathematics. Suffice it to recall a well-known problem called "The Problem Perps". At the end of the 20th century in 1995 was published in the prestigious Journal Annals of Mathematics erroneous work [2] for which the author A. Wiles received a dozen prestigious prizes including the prize of Abel Norwegian Academy of Sciences allegedly for a stunning proof of Fermat's theorem which tried to prove more than 350 years the most eminent mathematicians encluding Euler.

It is surprising that the 6 experts who tested Wiles' work 
could not recognise fallacy although this was obvious to anyone familiar with Kummer's conclusion formulated in the 19th century: "the complete proof of Fermat's theorem was beyond the capabilities of existimg mathematical approaches.

In 2000 The Clay Mathematical Institute decided to take over the duties of D. Hilbert declaring the seven Millennium problems left previously unsolved. One of these problems, namely the problem of Cook's Institute ruled out as the author of present work this problem was solved in 2008 and the corresponding works in press [3-4], and Cook, formulating this problem was mistaken in pointing out that the problems from $\mathrm{P}$ are parts of the problems from NP, which incorrect since we proved [3-4], that $P \neq N P$. It seems to us that The Institute (Clay) is engaged in charity only for reason to pay less taxes to the state.

The main task considered in the article is to find the

condition primitive integer solution of the diophantine Pithagorean equation $x^{2}+y^{2}=z^{2}$. It is known [5] that for this purpose it is enough to find primitive solutions of $x$, $y$ such that $x$ is even and $y$ is odd. In this paper, in particular, we proved that the $z$ of a primitive solution is a Prime number of the form $4 k+1$. If we divide the set of all natural numbers into residue classes modulo 4 [6], then all primes full into residue classes $\overline{1}, \overline{3}$. It is known [7] from the remarks left by Fermat that all primes of their $\overline{1}$ are representable as the sum of two squares and primes of $\overline{3}$ are never representable as the sum of two squares. These statements were proved by us in [8] with the help of the axiom of descent. Using the descent axiom, it is also easy to prove that there are infinitely many primes of the form $4 k+1$ and many of other problems of theory of numbers [9-15]. If the set of all natural numbers is divided into residue classes modulo 5 , then all primes fall into residue classes $\overline{1}, \overline{2}, \overline{3}, \overline{4}$. In class $\overline{1}$ are all Prime numbers end with 1 , in class $\overline{2}$ will be one Prime 2 and all primes ending in 7 , class $\overline{3}$ are all Prime numbers ending in 3 and in class $\overline{4}$ will be all primes with the end 9. With the help of the axiom of descent it is easily proved that there are infinitely many primes with endings $1,3,7,9$. Thus, this statement is a generalization of Euclid's fundamental theorem on the infinity of the set of Primes.

All twin numbers are in residue classes $(\overline{1}, \overline{3}),(\overline{2}, \overline{4}),(\overline{4}, \overline{1})$ and there are infinity many twins [15].

\section{Primitive Solutions of Diophantine Equation of Pythagor}

We consider the Pythagorean Diophantine equation

$$
\mathrm{u}^{2}+\mathrm{v}^{2}=\mathrm{w}^{2}
$$

Theorem. If $w$ in equation (1) is a Prime number of the form $4 \mathrm{k}+1$ then there is an even number $\mathrm{u}$ and an odd number $\mathrm{v}$ satisfying equation (1).

Evidence, The smallest natural number of the form $4 \mathrm{k}+1$ is 5 which is obtained when $\mathrm{k}=1$. Equation (1) for $\mathrm{w}=5$ will take the form $u^{2}+v^{2}=25$. If $u=4$, then for $v$ we get an odd number 3 .
Take as $\mathrm{w}$ the following Prime number of the form $4 \mathrm{k}+1$ equal to 13 at $\mathrm{k}=3$. Equation (1) for $\mathrm{w}=13$ will take the form $\mathrm{u}^{2}+\mathrm{v}^{2}=169$. If $\mathrm{u}=12$, then we get $\mathrm{v}^{2}=25$, where for $\mathrm{v}$ we get an odd number 5 .

Suppose that $\mathrm{w}$ is a Prime number $\mathrm{n}$ of the form $4 \mathrm{k}+1$ then from equation (1) for $u$ we get an even number $u_{n}$ and for $\mathrm{v}$ an odd number $\mathrm{v}_{\mathrm{n}}$.

Let now for a Prime number $n^{\prime}>n$ of the form $4 k+1$ from equation (1) there are no numbers $u$ and $v$ satisfying this equation. Then according to the descent axiom for the number $\mathrm{n}$ there are also no numbers $\mathrm{u}$ and $\mathrm{v}$ satisfying equation (1) and this contradicts our inductive assumption. Hence the statement of the theorem.

Corollary. A Prime number $\mathrm{P}$ is The Hypotenuse of an integer right triangle if and only if $\mathrm{P}-1$ is divisible by 4 .

Evidence. Indeed, it follows from the proof of the theorem That the hypotenuse of an integer right triangle of length $\mathrm{P}$ will be if and only if when $P$ is a Prime number in form of $4 \mathrm{k}+1$ and $\mathrm{P}-1$ is divisible by 4 .

\section{Primitive Solutions of the Pythagorean Equation for Two Mutually Prime Numbers of Different Parity}

It is known from [5] that if $\mathrm{m}$ and $\mathrm{n}<\mathrm{m}$ are mutually Prime numbers of different parity, then the formulas (2) deliver some primitive solution of equation (1) with even $\mathrm{x}$; Back any consisting of positive integer primitive solution of equation (1) for which $\mathrm{x}$ is even numbered by formulas (2), where $\mathrm{m}$ and $\mathrm{n}<\mathrm{m}$ mutually prime numbers of different parity

$$
\begin{gathered}
\mathrm{x}=2 \mathrm{mn} \\
\mathrm{y}=m^{2}-n^{2} \\
\mathrm{z}=m^{2}+n^{2}
\end{gathered}
$$

So, for example, if we take $\mathrm{m}=9, \mathrm{n}=4$, then $\mathrm{x}=2 \mathrm{mn}=72$, $y=m^{2}-n^{2}, z=m^{2}+n^{2}$ is a primitive solution of equation (1).

Thus we find all primitive solutions of equation (1), when the hypotenuse of a right triangle is a Prime number of the form $4 \mathrm{k}+1$ and primitive solutions of the Pythagorean equation for two given mutually Prime number $m$ and $n<m$ of different parity. Obviously, when we look for a primitive solution of the Pythagorean equation using the formulas (2), the hypotenuse $\mathrm{z}$ of a right triangle is equal to $m^{2}+n^{2}$. Therefore, the formulas (2) allow us to find primitive solutions $(\mathrm{x}, \mathrm{y}, \mathrm{z})$ of the Pythagorean equation if we take the number 4 as $n$, and any Prime number ending in 3 or 7 as $m>4$. In this case, obviously, the hypotenuse of a right triangle will be a compaund number ending in 5.

\section{Conclusions}

All triples of natural numbers $(x, y, z)$ of primitive solutions of Diophantine Pithagorean equation (1) can be divided into two classes: one class include all triples in which $\mathrm{z}$ is a Prime of the form $4 \mathrm{k}+1$ and the other class include triples for which $\mathrm{z}$ 
is not a Prime of the form $4 \mathrm{k}+1$. However in all triples of primitive solutions of equation (1) $\mathrm{x}$ will be even and $\mathrm{y}$ will be odd.

The first class of primitive solutions of equation (1) include right integer triangles with hypotenuse the lengths of which are Prime numbers of form $4 \mathrm{k}+1$ and the second class right integer triangles with hypotenuses the lengths of which are composite numbers for example obtained from mutually prime numbers of different parity: (4, Prime number ending at $3 \_$) ou (4, Prime number ending at 7).

\section{References}

[1] Kochkarev B. S. K metodu spuska Ferma. Problems of Modern Science and Education, 2015, 11 (41), pp. 7-10 (in Russian).

[2] Wiles A. Modular elliptic curves and Fermat's last theorem Annals of Mathematics, v, 141 Second series, 1995, pp. 445-551.

[3] Kochkarev B. S. Problem of Recognition of Hamiltonian Graph. International Journal of Wirless Communication and Mobile Computing 2016, 4 (2), 52-55.

[4] Kochkarev B. S. Vzaimootnosheniya mejdu slojnostnymi klassami P, NP i NPC. Problems of Modern Science and Education, 2015, 8 (35), 6-8. (in Russian).

[5] Postnikov M. M. Vvedenie v teoriyu algebraicheskich chisel. Nauka, 1982 s. 240 (in Russian).
[6] Buchshtab A. A. Teoriya chisel. Prosvetshenie. 1966 s. 384 (in Russian).

[7] Singh S. Velikaya teorema Ferma. MTSHMO, 288 \{in Russian).

[8] Kochkarev B. S. Problema bliznetsov i drugie binarnye problemy. Problems of Modern Science and Education. 2015, 11 (41), s. 10-12. (in Russian).

[9] Kochkarev B. S. About One Binary Problem in a Class of Algebraic Equation and Her Communication with the Great Hypothesis of Fermat. IJCMS, v. 2, Issue 10, pp. 457-459, October 2016.

[10] Kochkarev B. S. Infinity Sequences of Prime of Form 4n-1 and $4 n+1$, International Journal of Humanities and Social Science Invention, v. 5, Issue 12, December 2016.

[11] Kochkarev B. S. Algorithm of Search of Large Prime Numbers. International Journal of Discrete Mathematics, 2016, 1 (1) pp. 30-32.

[12] Kochkarev B. S. An Binary Problem of Goldbach Euler and its Generalization. International Journal of Discrete Mathematics, 2918, 3 (2), pp. 32-35.

[13] Kochkarev B. S. About Tenth Problem of D. Hilbert. AJER, Vol. 6, Issue, 12, 2017, pp. 241-242.

[14] Kochkarev B. S. Axiom of Descent and Binary Mathematical Problem, AJER, 2018, Vol. 7, Issue, 2, pp. 117-118.

[15] Kochkarev B, S. Zakonomernosti generatsii prostykh chisel i prostykh chisel bliznetsov. International Journal Chronos, 3 November, 2018, 52-53 (in Russian). 